\title{
Current Strategies and Potential Prospects of Nanomedicine-Mediated Therapy in Inflammatory Bowel Disease
}

\author{
Fengqian Chen' \\ Qi Liu ${ }^{2}$ \\ Yang Xiong ${ }^{3}$ \\ $\mathrm{Li} \mathrm{Xu}{ }^{4}$
}

'Translational Research Program, Department of Anesthesiology and Center for Shock Trauma Anesthesiology Research, University of Maryland School of Medicine, Baltimore, MD, 2I20I, USA; ${ }^{2}$ Department of Dermatology, Johns Hopkins University School of Medicine, Baltimore, MD, 2123I, USA; ${ }^{3}$ College of Pharmaceutical Science, Zhejiang Chinese Medical University, Hangzhou, Zhejiang, 310053, People's Republic of China; ${ }^{4}$ Department of Anorectal Surgery, The First Affiliated Hospital of Zhejiang Chinese Medical University (Zhejiang Provincial Hospital of Traditional Chinese Medicine), Hangzhou, Zhejiang, 310006,

People's Republic of China
Correspondence: Li Xu Email200530I2@zcmu.edu.cn

\begin{abstract}
Inflammatory bowel diseases (IBD) such as Crohn's disease and ulcerative colitis are highly debilitating. IBDs are associated with the imbalance of inflammatory mediators within the inflamed bowel. Conventional drugs for IBD treatment include anti-inflammatory medications and immune suppressants. However, they suffer from a lack of bioavailability and high doseinduced systemic side effects. Nanoparticle (NP)-derived therapy improves therapeutic efficacy and increases targeting specificity. Recent studies have shown that nanomedicines, based on bowel disease's pathophysiology, are a fast-growing field. NPs can prolong the circulation period and reduce side effects by improving drug encapsulation and targeted delivery. Here, this review summarizes various IBD therapies with a focus on NP-derived applications, whereas their challenges and future perspectives have also been discussed.
\end{abstract}

Keywords: inflammatory bowel disease, nanoparticles, targeted delivery, nanomedicine applications

\section{Introduction}

Inflammatory bowel disease (IBD) is an umbrella term of immune responsemediated chronic remission and relapse bowel diseases. IBD is classified with Crohn's disease (CD) and ulcerative colitis (UC) with different etiologies. CD and UC are diseases of unidentifiable causes. CD presents ulcers and granuloma centered on the small and large intestines where inflammatory occurs in the alimentary canal from the mouth to the anus. UC presents inflammatory response and successive ulcers and abscesses in the colonic mucosa. ${ }^{1,2}$ Patients with IBD have a raised potential risk relative to colon cancer. Due to the chronic inflammation development and a great number of immune cell filtration as well as immune cell-mediated organ destruction, IBD has become the third most common disease worldwide. $^{3}$

IBD is a chronic inflammatory syndrome that influences the gastrointestinal (GI) tract and shows clinical variations not only in the developed countries (such as North America and Europe) but also in the developing countries (such as India and China). ${ }^{4,5}$ More than 5,000,000 people globally suffered from IBD. Currently, approximately 25 per million people yearly (developed countries) and 5 per one million people yearly (developing countries) lived with. ${ }^{6}$ This chronic inflammatory and debilitating disease need lifetime therapy, with enormous financial burden and healthcare system support. ${ }^{7}$ 
Colitis-associated colorectal cancer and sporadic colorectal cancer are mostly developed due to two significant issues: irregular inflammation and immunosuppression of carcinogenesis. ${ }^{8}$ Recent research has shown that chronic inflammation in IBD could trigger the development of colorectal cancer. Oxidative stress from inflammation sites can induce DNA damage, leading to the activation of pro-carcinogenic genes or the silence of tumor suppressor signals. Moreover, microbiota alteration in the gut can lead to chronic inflammation-mediated carcinogenic component productions. These mechanisms are attracting increasing research interests. ${ }^{9}$

Recently, fast-developing diagnosis and treatment in IBD, such as the use of anti-inflammatory agents, have significantly reduced the surgery and hospitalization rates for patients. Therapies such as 5-aminosalicylic acid, corticosteroids, immunomodulators, antibiotics, and biological agents have been widely offered in IBD treatment, significantly reducing colitis-associated colorectal cancer. ${ }^{10-12}$ These tools are also defined as effective early preventions. However, immunomodulators may often lead to severe side effects among healthy tissues, such as lymphoma development. ${ }^{13}$ Without targeting delivery, IBD treatment drugs may be absorbed into the systemic circulation and exposure to healthy tissues, resulting in increased severe side effects. Drug efficacy and safety have become an emerging question that calls for advanced drug delivery systems that would target-deliver medications into the inflamed sites and avoid absorption from the healthy tissues. ${ }^{14}$

Nanomedicines have been widely utilized in the pharmaceutical field for loading hydrophobic drugs, showing a significantly reduced dosage use and increased treatment efficiency, leading to promisingly minimized systemic side effects. ${ }^{15,16}$ Current conventional therapeutic agents, such as powders and tablet formulations (orally administrated) as well as solution and emulsion formulations (intravenously injected), are widely used for improving drug bioavailability. ${ }^{17}$ Nanocarriers may facilitate such conventional delivery systems in a way that could overcome existing biological barriers, thus delivering drugs to designed specific sites. ${ }^{18,19}$ In the following content, we will focus largely on such new strategies that the nanocarrier system could bring in for IBD treatments. ${ }^{20,21}$

\section{Disease Pathogenesis}

In a closer look, IBD is a consequence of the dysregulated immunology among the chronic damaged of inflamed gut against antigens activation. ${ }^{22}$ In specific, bacterial load and alpha diversity significantly decreased in the microbiome environment, with a change of $\mathrm{pH}$ ranging from 2.3 to 8.3 within $\sim 24 \mathrm{~h}$ transit time frame. ${ }^{23,24}$ Compared to healthy bowel, IBD induces multiple unwanted symptoms such as fever, abdominal pain, bowel obstruction, chronic diarrhea, rectal bleeding, and weight loss, eventually causing colorectal cancer. The development and pathogenesis of IBD are multifactorial, representing a complex reciprocation between different elements such as environmental factors, microbial dysbiosis, and genetic variations. ${ }^{25,26}$

When IBD occurs, innate immunity is the first line to initiate a primary defense by neutrophil accumulation and antigen activation, and then mononuclear phagocyte infiltration. ${ }^{27}$ In a normal homeostatic stage, macrophages and dendritic cells could effectively recruit TNF- $\beta$ and IL10 -secreting $\mathrm{T}$ regulatory cells to sites to prevent imbalanced inflammation. The activated antigen presentation cell, such as dendritic cells, could provide antigens to activate naive $\mathrm{T}$ cells into effector cells. But this is not the case during IBD. ${ }^{28,29}$

Physical barriers and chemical barriers both changed in the inflamed tissue, resulting in epithelial barrier integrity loss and immune cell (such as Th1, Th2, and Th17) recruitment. Functional reprioritizing of leukocytes and leukocyte-mediated "genetic storm" have been widely studied in IBD. ${ }^{30}$ Such studies showed that immune activation during IBD arouses protective effects, balancing the excessive amount of pro-inflammatory and antiinflammatory factors. These factors are dynamically mediated by the production of targeted chemotaxis, cytokines, and the generation of reactive oxygen species (ROS), phagocytosis, nitric oxide, and matrix metalloproteases. ${ }^{31}$ The systemic inflammatory responses expanded into numerous immune system-activating aspects, as well as considerably suppressive aspects. Although naturally developed to clear molecular dangers, these extensively generated pro-inflammatory cytokines (such as IFN- $\gamma$, IL-17, IL-4, and IL-13) may often facilitate IBD pathogenesis. ${ }^{32}$

\section{Current Therapeutics}

Traditional therapeutic approaches often function on mediating the systemic immune responses via regulating mucosal and aggravated inflammatory progression. These therapeutics are based on immune-modulatory medicines (such as aminosalicylic acid, corticosteroids, immunomodulators (6-Mercaptopurine and Azathioprine), antibiotics, 
and biological agents (Infliximab)), as well as strategies against pro-inflammatory factors (chemokines, integrins, and cytokines). ${ }^{33-37}$

For instance, corticosteroid dampens the initiation of the inflammatory response by hampering pro-inflammation cytokine production, inhibiting immune cell recruitment, and suppressing cellular translation of nuclear factor- $\kappa \mathrm{B}$ $(\mathrm{NF}-\kappa \mathrm{B})$. As a result, it improves vascular permeability, vasodilation, neutrophil infiltration, fibroblast activation, vascular proliferation, and collagen deposition. ${ }^{38,39}$ Immunosuppressive medicines, such as tacrolimus, ${ }^{39}$ mercaptopurine, ${ }^{40}$ methotrexate, ${ }^{41}$ azathioprine, ${ }^{42}$ and cyclosporine, ${ }^{43}$ could effectively inhibit lymphocytes activation and proliferation. Amino salicylate, for example, suppresses macrophage chemotaxis, mitogen-activated protein (MAP) kinase, and NF- $\kappa \mathrm{B}$ signaling pathways. It also increases the peroxisome proliferator activator receptor gamma (PPAR $\gamma){ }^{44}$

The dysfunction of the intestinal immune system may lead to microbial dysbiosis in the gut microbial community, resulting in IBD. A variety of therapeutics is applied for immune dysregulation. For initial therapy and remission, antibiotics (such as ciprofloxacin and rifaximin) and probiotics (such as Bifidobacterium and Lactobacillus) are applied. $^{45}$ One of the vital biological treatments in IBD is monoclonal antibodies (mABs). ${ }^{46}$ The mABs against proinflammation cytokines such as TNF- $\alpha$ and IFN- $\gamma$ would target crosstalk among IL-23, IL-6, IL-17, IL-13, and TGF- $\beta$. The mABs against adhesion molecules like ICAM-1 could inhibit the recruitment of effector T cells. ${ }^{4,48}$ Inhibitors against chemokine receptors, homing-linked receptors (such as $\alpha 4 \beta 7$ integrin), and sphingosine-1-phosphate receptors have also shown promising effects on IBD treatment. ${ }^{49,50}$ Notably, in the clinic, current large-scale applications for IBD treatment include infliximab (anti-TNF- $\alpha$ mAbs), ${ }^{51}$ vedolizumab ( $\alpha 4 \beta 7$ integrin inhibitor), ${ }^{52,53}$ ustekinumab (IL12/IL-23 inhibitor), ${ }^{52,54}$ and tofacitinib (JAK inhibitor). ${ }^{55}$

Immune cells are also largely investigated in IBD treatment. Cell-based therapies often include the suppression of immune response associated with antigenpresenting cells (macrophages and dendritic cells) and effector $\mathrm{T}$ cells, and also the depression of proinflammatory cytokines produced by leukocytes. ${ }^{28,56}$ Recently, mesenchymal stem cells and hematopoietic stem cells are regarded as potential treatments for IBD. ${ }^{57-61}$ These cells have shown cell renewing property and may differentiate into multiple cell types with immune-suppressive and anti-inflammatory effects, as well as long-term gene expression tendency.

Although comparably effective, conventional treatments for IBD are limited in many aspects, such as minimal drugbioavailability and systemic drug exposure-induced side effects. ${ }^{62,63}$ Nowadays, a fast-growing field of IBD treatment is focusing on developing new therapeutic strategies with various drug delivery systems. These systems may help us gain insights into disease progression and prognosis. ${ }^{63,64}$

\section{Nano-mediated Therapies}

In 2001, the targeted accumulation of small nanoparticles (NPs) size around $100 \mathrm{~nm}$ in the inflamed murine colon rather than a healthy murine colon was first demonstrated. ${ }^{65,66}$ Because of this passive accumulation property, NPs have become a promising drug delivery system for IBD treatment. Defective mucus layer and loss of barrier integrity of the inflammatory intestinal mucosa enable NPs to leak into the inflamed tissue passively. In specific, the defective epithelial barrier of inflamed intestinal tissue would increase gut barriers' permeability, which promotes NPs' infiltration. Moreover, inflamed mucosa increases mucus secretion, which facilities NPs' adhesion and diffusion through the layer of intestinal mucus. In the meantime, immune cells such as neutrophils and macrophages also infiltrate into the layer of inflamed mucosal and submucosal, promoting cellular uptake of NPs to the inflammation sites. ${ }^{15,67,68}$

Accumulated research has shown the safety and effectiveness of nanomedicines as a promising approach for IBD treatment. With the help of rational drug delivery systems, conventional treatment drugs can be packed into the inflammatory tissues to treat IBD. Not only delivering natural compounds and conventional agents, but these delivery systems can also facilitate remission of IBD by enhancing treatment efficacy and reducing systemic exposure in the healthy tissues. ${ }^{69-71}$ On the one hand, these delivery systems had passively or actively target themselves to the inflammation sites. On the other hand, they increase drug bioavailability and concentrations. Owing to epithelial enhanced permeability and retention effect (EPR), small size NPs were selectively uptake into the inflamed tissues' intracellular matrix. ${ }^{72-74}$ Increased permeability of the intestinal epithelial barrier due to IBD also facilitates the transcellular transport of NPs. Once uptake, endocytosis induces NPs to transcytosis at the apical membrane of cells. NPs are then released at the 
basolateral pole and interact with leukocytes at the submucosal layer. ${ }^{75-79}$

By increasingly understanding pathophysiological and pathogenesis in the inflammatory GI tract, we herein review the current nanocarriers attributed to inflammatorytargeted drug delivery.

\section{Nanocarrier Systems \\ Biodegradable Nanomedicines}

Drug delivery systems aiming to protect the hydrophobic agents often work by avoiding premature degradation and enhancing the sustained release of drugs at the targeted site. ${ }^{80}$ A controlled-release system, in such cases, provides an ideal strategy to maintain the frequency and concentration of drug release. ${ }^{73,81-83}$

For instance, high-water content hydrogel, a copolymer with a cross-linked network, has high biocompatibility with its physical similarity to biological tissues. ${ }^{84}$ Hydrogels are proven effective in loading hydrophilic drugs and protecting them from being desaturated or aggregated. $^{85}$ It has been shown that a hydrogel drug delivery system with alginate and chitosan in encapsulating anti-inflammatory tripeptide Lys-Pro-Val (KPV) to be significantly effective. Upon delivering to the inflamed colon, the hydrogel was efficiently released for treating colitis disorder in this dextran sulfate sodium-induced colitis model. ${ }^{86}$

Lipid NPs packaged with budesonide, when orally administrated, have shown a significant antiinflammatory effect in colitis in vivo compared to free drug usage. After 12 hours of administration, a significantly higher concentration of NPs was detected in the inflamed colon. Alongside, these lipid NPs also improved histological scores, reduced myeloperoxidase activity, and suppressed pro-inflammatory cytokines in the inflamed colon. ${ }^{87}$ On the other hand, hyaluronic acidconjugated self-assembled NPs, when loaded with budesonide, have also shown a significant suppression of TNF and IL-8 among inflamed cells in vitro. ${ }^{88}$ In these studies, NPs are proven as biocompatible vehicles with minimal toxicity. As hyaluronic acid showing an anti-inflammatory effect, blank NPs also elicited significant IL-8 reduction. ${ }^{88}$

In rodent colitis models, dexamethasone-loaded solid lipid nanoparticles, when orally administrated, could significantly suppress pro-inflammatory cytokines IL-8 and TNF compared to the free drug. ${ }^{89}$ Orally administered the Immunosuppressant tacrolimus-loaded PLGA NPs when orally administrated have also shown significantly higher drug accumulation in the inflamed tissue and greater colitis resolution than standard formulations in comparison. ${ }^{90}$

When incorporated into NPs, anti-sense oligonucleotides could achieve high stability by NPs' protection away from DNases' degradation. In a rodent colitis model, orally administrated NF- $\mathrm{KB}$ anti-sense oligonucleotides showed efficacy. ${ }^{91}$ In the clinic, anti-sense oligonucleotide treatments such as Alicaforsen and Mongersen are also involved. ${ }^{92,93}$ These treatments are believed to be beneficiaries of the NP-mediated drug delivery system. ${ }^{78,94-96}$ Moreover, recombinant protein prohibitors-loaded PLGANPs, when orally administrated, have shown significant antioxidant effect and suppression of colitis disease severity. $^{97}$

TNF is one of the vital targets for IBD treatment which plays an essential role in disease immunity. ${ }^{98}$ It originates from innate immune cells, feedbacks between innate and adaptive immune systems, and dominates IBD pathogenesis. In the mice model, intraperitoneal administration of engineered NPs loaded with polyethyleneimine/TNF- $\alpha$ siRNA had significantly suppressed TNF- $\alpha$ expression compared to siRNA itself. ${ }^{99,100}$

\section{Natural Product-based Nano-systems}

Natural polysaccharides, such as pectin, alginate, and chitosan, have been developed as oral hydrophobic drug delivery systems for targeting inflamed colon. These systems are non-toxic, easily manufactured, and FDA approved. These applications from the polysaccharide family protect against premature drug release and selectively release IBD drugs into the small intestine, colon, and also stomach. $^{101,102}$

For instance, an engineered polysaccharide system significantly suppressed colitis by inhibiting IL-22 expression. ${ }^{103}$ Eucheuma cottonii and Acmella oleraceaextracted polysaccharides have been shown to regulate inflammation and inhibit colonic damage in dextran sulfate sodium (DSS)-induced colitis. ${ }^{103,104}$ Non-starch polysaccharides were used for IBD treatment in vitro and in vivo, exhibiting significant improvement of immune stimulation, gut-microbiota modulation, anti-inflammation, and reoccurrence rates. ${ }^{105}$ Amylose cornstarch packaged with Mesalamine has shown an accurate release of drug in the inflamed colon with a high withstandment of enzymatic and acidic conditions in the GI tract. ${ }^{106}$

Poly (epsilon-caprolactone) (PCL) microspheres, consisting of NPs-in-microparticles, were designed to load plasmids and nucleic acids in oral deliveries. These 
microspheres have been shown to target released at the inflamed intestine and tolerance against enzymatic degradation in the upper GI tract. ${ }^{107,108}$ Moreover, quercetinloaded chitosan-based NPs have been reported in an acetic acid-induced colitis rabbit model, demonstrating effectiveness compared to free drug. ${ }^{109,110}$

Edible plant-derived non-toxic NPs have also been studied for IBD treatment. ${ }^{111}$ Grapefruit-derived NPs have the capacity of biodegradable, biocompatible, and stable in various $\mathrm{pH}$ conditions. Methotrexate-loaded grapefruit-derived NPs upgraded anti-inflammatory response in dextran sulfate sodium-induced IBD by reducing IL- $1 \beta$ and TNF- $\alpha$ in macrophages of intestine tissue. ${ }^{112}$ Ginger-derived NPs have been developed to increase intestinal epithelial cell proliferation by upregulating anti-inflammatory cytokines and reducing proinflammatory cytokines (IL-6, TNF- $\alpha$, and IL-1 $\beta$ ). ${ }^{113}$

Phytochemicals can target various pathogenesis and inflammation routes in IBD. A natural biopolymer-based nanocarrier is designed to carry an active compound 6-shogaol to the inflamed colon. ${ }^{101,114}$ Ulva lactuca polysaccharide-selenium NPs represent a promising therapeutic approach in anti-inflammatory for acute colitis. ${ }^{115}$ Broccoli-extracted NPs demonstrated suppression of colitis by stimulating adenosine monophosphate-activated protein kinase. ${ }^{116}$ In another study of the colitis model, krill oil liposomes loaded with budesonide could also inhibit TNF- $\alpha$ and have shown great potential in oral drug delivery. ${ }^{117}$

\section{Cells and Exosomes}

Bioengineered red blood cells as nanoparticle carriers is a new strategy for IBD treatment, showing high biocompatibility capacities and a significantly longer circulation time within blood. ${ }^{118,119}$ In a clinical trial among pediatric steroid-dependent CD patients, autologous red blood cells were used to load dexamethasone-21-phosphate, showing a significant therapeutic effect with $44 \%$ remission in patients. ${ }^{120}$ In the next six years' continued study, 50\% of patients showed sustained therapy efficacy and safety. ${ }^{121,122}$

Exosomes are extracellular vesicles (EVs) that are released by mammalian cells. Due to their nano-sizes, these EVs are considered natural NPs. EVs can transport signals such as proteins and RNAs from host cells to the recipient cells. ${ }^{123}$ EVs have been used for various innovative drug delivery strategies so far. For instance, intestinal exosomes have been proven as a new strategy for IBD therapy. ${ }^{124,125}$ Intestinal epithelial cell-derived EVs contain a large amount of transforming growth factor-beta 1 (TGF- $\beta 1$ ) with immunosuppressive activity. ${ }^{126}$ In dextran sulfate sodium-induced colitis, these EVs worked on immunosuppressive dendritic cells and regulatory $\mathrm{T}$ cells to protect against colitis progression. In an in vivo model, EVs secreted by granulocytic myeloid-derived suppressor cells were demonstrated to reduce dextran sulfate sodiuminduced colitis, showing an attenuated Th1 cell population and an enhanced $\mathrm{T}$ regulator cell population in mesenteric lymph. ${ }^{127}$

Reduced levels of interferon (IFN)- $\gamma$ and TNF- $\alpha$ were found in mice models of IBD. A study exhibited that IL-10 incubated bone marrow-derived DCs would secret EVs to reduce colitis from trinitrobenzene sulfonic acid induction. In specific, EVs from IL-10 treated cells were associated with IL-10 mRNA upregulation, IL-2, IFN- $\gamma$, and TNF- $\alpha$ mRNAs downregulation, and $\mathrm{T}$ regulator cell upregulation in colonic tissue. These studies implied the importance of EVs as potent natural nanoparticles for transporting biological contents in IBD treatment. ${ }^{128}$

The repairs of injured cells/tissues in the intestine can also benefit IBD treatment. Mesenchymal stem/stromal cell-derived EVs have shown therapeutic efficacy on tissue injury repair in colitis. ${ }^{129,130}$ EVs secreted by mesenchymal stem/stromal cells in bone marrow, adipose tissue, and the umbilical cord could provide a promising approach for regenerative therapy. These EVs have the ability to migrate into damaged tissues, promote tissue repair, and modulate immune responses. ${ }^{20,129,131}$ Therefore, EVs may serve as potential nanocarriers for efficient, targeted drug delivery in IBD treatment.

\section{Targeted Drug Delivery System}

\section{Targeted Nano-systems}

Depending on the inflammation level (luminal or systemic) and severity (mild, moderate, or severe) of IBD, nano-delivery systems can be administrated either orally or intravenously. ${ }^{122}$ Luminal and mild inflammation can be nano-targeted orally, whereas severe systemic inflammation often requires targeted intravenous delivery. In IBD, various nano-systems have adapted different targeting strategies to enhance inflammation site-delivery. These strategies include but not limited to passive targeting NPs; mannose receptor targeting NPs; inflammatory receptor targeting NPs; charge-mediated targeting NPs; and microenvironment-responsive targeting NPs (eg, redox, ROS, and $\mathrm{pH}$-triggered release). ${ }^{62,132}$ 
Various receptors are overexpressed in the endothelium of the inflammatory bowel. Therefore, ligand-mediated NPs may target these receptors and increase the accumulation of drugs in the inflamed sites. ${ }^{133,134}$ For instance, hyaluronic acid could precisely target to CD44 receptor. Overexpressed CD44 on the surface of inflamed macrophages and epithelial cells can be specifically bond to hyaluronic acid among colitis tissues. ${ }^{135,136}$ On a UC mice model, hyaluronic acid-incorporated copolymeric NPs were encapsulated with tripeptide for colitis delivery. ${ }^{137}$

Inflammatory sites also exhibit an overexpressed level of folate receptors. Folate receptor-targeted NPs have been demonstrated to bind to activated macrophages and accumulate in the inflamed intestine in mice colitis via activemediated targeting. ${ }^{114,138,139}$ Moreover, macrophages express abundant mannose receptors in the membranes. An ex vivo study has shown that mannosylated-target PLGA-PEG NPs had more accumulation of model drug ovalbumin in inflamed murine colonic tissues than nontargeted PLAG-PEG NPs. ${ }^{15}$

Mesalazine-loaded pectin-silica-based NPs were designed to be controlled-release in the colon. These NPs exhibited a minimized release in the upper GI tract but increased release of mesalazine in the simulated colonic fluid due to enhanced sensitivity of pectin towards the pectinase. ${ }^{140}$ On the other hand, TNF siRNA loaded macrophage-targeted copolymer NPs effectively targeted macrophages and increased therapeutic effect than nontargeted NPs in IBD treatment. In rodent colitis models, the combination of TNF and cyclin D1 siRNA-loaded multi-compartmental NPs-in-microsphere oral system showed lipase-triggered drug release in the intestine. It exhibited significant gene silencing, suppression of myeloperoxidase activity, and also IL-1 and IFN reduction. ${ }^{141}$

CD98 plays a vital role in the homeostasis of the intestinal immune responses. It is overexpressed in epithelial cells and macrophages in the colon of IBD. ${ }^{142}$ In the mice model, upregulated CD98 leads to colitis-associated tumorigenesis. In the dextran sodium sulfate-induced mice model, CD98 siRNA-coated polyethyleneimine (PEI)/ polylactic acid NPs were orally administered to treat colonic inflammation. ${ }^{143}$ The results showed a significant suppression of $\mathrm{CD} 98$ protein in the macrophages and epithelial cells of the intestine. In another rodent colitis model, this NPs-mediated drug delivery system has also been used for the local delivery of IL-10 expressing plasmid. Their results showed significant suppression of inflammation, and highly improved clinical activity score. ${ }^{143,144}$ Thus, ligand-incorporated NPs are demonstrated effective in various applications.

\section{The Trigger-release Nano-systems}

Resveratrol is a hydrophobic therapeutic agent from a natural herb for the treatment of IBD. A study has shown that a $\mathrm{pH}-$ trigger-released poly $(\mathrm{N}, \mathrm{N}$-dimethylamino ethyl methacrylate) NP system was established. In this system, chitosan was integrated into a hydrophilic vector, showing high biocompatibility and low toxicity. The loaded resveratrol was sustain-released for drug efficacy. ${ }^{145}$

Eudragit-coated budesonide-loaded PLGA NPs were $\mathrm{pH}$-dependent in ameliorating murine colitis model and demonstrated significantly increased efficacy compared to non-coated NPs. Eudragit loaded PLGA-modified copolymeric blend. Eudragit ${ }^{\mathbb{R}} \mathrm{L}$ and Eudragit ${ }^{\mathbb{B}} \mathrm{S}$ are anionic copolymers containing methacrylic acid and methyl methacrylate and are approved non-biodegradable by FDA. These non-absorbable, non-toxic, and $\mathrm{pH}-$ dependent polymers are often utilized for IBD treatment. ${ }^{146}$ Moreover, dual sensitive ( $\mathrm{pH} /$ time, $\mathrm{pH} /$ enzyme, etc.) release NPs have also been developed to improve IBD therapy. For instance, carboxymethyl inulin responds to both redox and $\mathrm{pH}$. Carboxymethyl inulinengineered NPs showed a high accumulation in the inflamed tissue than that of free drug in colitis mice. ${ }^{147}$

The concentration level of endogenous ROS indicates oxygen metabolism. Increased ROS was found in the inflammatory intestinal mucosa. In IBD patients, mucosal ROS concentration is $10-100$ times higher in the inflamed intestine than health controls. ${ }^{148,149}$ Nitroxide radicals in NPs have been shown to selectively and effectively inhibitions ROS. In a colitis model, the disease activity index was significantly lower after seven days of orally administering engineered NPs. ${ }^{150}$ In another study, NPs were designed to trigger release molecules' free radical scavenger tempol to increase ROS concentrations in inflamed intestinal sites. Their design exhibited high therapeutic efficacy in both acute and chronic colitis mice. ${ }^{151}$

Orally administered nucleic acid for IBD therapy is usually hindered by poor cell membranes trans passing. NPs, when logically designed, may enhance RNA payload delivery. For instance, the pro-inflammatory cytokine TNF plays an important role in the progression and development of IBD. A recent study found that orally administrated, TNF siRNA-loaded Thioketal NPs may degrade in an environment of high ROS concentration in the inflamed 
sites and may successfully suppress colonic TNF protein expression in colitis in vivo. ${ }^{150,152}$

\section{The Charge-mediated Targeting}

In IBD, the charged surface of inflammatory tissues can be specifically targeted by NPs of opposite charge via electrostatic interactions. The mucosal composition of the inflamed colonic mucins comprises negatively charged carbohydrate fractions, unlike the healthy regions. Thus, cationic NPs as nanocarriers can increase mucous interaction and prolong drug residency. ${ }^{153-155}$ In ex vivo studies, researchers have demonstrated significantly increased targeting efficiency of positively charged chitosan NPs to the human inflammatory intestinal mucosa. ${ }^{156}$

On the other hand, cationic proteins such as transferrin and eosinophil cationic proteins are abundant in inflamed areas. Thus, negatively charged NPs give preferential adhesion to these cationic proteins. In a colitis model of rats, negatively charged liposomes exhibited a higher accumulation in the inflammatory regions than cationic or neutral charged liposomes. ${ }^{157}$ These results showed that NPs positively or negatively charged can interact with components in the GI tract and offer specificity in drug deliveries. ${ }^{87}$ However, unwanted electrostatic interactions remain issues among these systems. Charged NPs may interact with the oppositely charged GI tract components like soluble mucins and bile acids. Thus, the efficacy remains investigated. The combination of ligand-mediated and charge-mediated NPs may facilitate the specificity. ${ }^{158-160}$

\section{Conclusion and Future Perspectives}

Current drugs for IBD, such as 5-aminosalicylic acid derivatives, corticosteroids, anti-TNF mediators, immunosuppressive agents, and other biologic components, suffer from immunosuppression and long-term systemic exposure. The utilization of nanocarriers could provide an efficient and safe approach for IBD therapy. To date, various nanocarriers have been studied, including selfassembled polymeric NPs, natural product-based NPs, exosomes, etc.

Compared with the conventional method, nanocarriers may prolong the retention period at inflamed sites and minimize administration frequency. Moreover, with the help of specific ligands-conjugated NPs, therapeutical agents can be delivered and accumulated to the inflamed GI tract, showing both high drug concentration at the disease sites and minimal systemic exposure to healthy tissues. Here we summarized recent research using nanodelivery strategies for IBD treatment. These delivery systems have been studied in multiple types of research and clinical trials with satisfactory results.

In summary, inflammatory region-specific targeting nanocarriers are likely to present a promising result in IBD treatment. The orally administrated formulation is the most desirable route so far, giving a suitable alternate for parenteral administrations. The versatility design of nanocarriers for proteins and nucleic acids also opens up opportunities for more advanced drug delivery designs, such as charge-mediated targeting, micro-environment triggered release targeting, and ligand-mediated targeting. These advanced drug delivery systems have shown the potential to improve current IBD treatment with enhanced therapeutic efficacy and improved patients' life qualities.

Although general concerns have been brought up on the level of nanocarriers' adverse effects, no factual data or conclusion has occurred so far. Nanotoxicology studies in animal models and the human GI tract are a must. NP sciences expand to various sizes, materials, and surface interactions. Thus, their applications in IBD therapy should be further explored with an increasing understanding of the human GI tract. Immune cells and immune functions altered dramatically during inflammatory diseases' pathogenesis. Major immune regulators such as macrophages polarized to a pro-inflammatory state, facilitating system dysfunction. Thus, the switch of immune cells' phenotypes with the help of NPs can be further investigated. With most nanocarrier designs applied in UC inflammation, therapies toward CD should be emphasized in the future.

The trend of applying multifunctional, multi-targeting NP in diagnosis and therapy is rising, but for successful clinical translation achieving long-term safety and minimizing pre-metabolism within system circulation are still the key. NP structural stability needs further optimization and validation. For NP scale-up and reliable manufacturing, delivery systems are often simplified from bench to bedside. Such simplification should base on achieving reduced drug load as well as prolonged remissions. Designing efficacious formulations and dosage forms for human administration needs to be further explored. Personalized patient IBD therapy tailored by targeted nanomedicine will be realized.

\section{Acknowledgments}

This study was supported by the National Natural Science Foundation of China, Grant No 81704082, and Medical 
Scientific Research Foundation of Zhejiang Province, China (Grant No.2020RC086).

\section{Disclosure}

The authors report no conflicts of interest in this work.

\section{References}

1. Molodecky NA, Soon S, Rabi DM, et al. Increasing incidence and prevalence of the inflammatory bowel diseases with time, based on systematic review. Gastroenterology. 2012;142(1):46-54. e42.

2. Kostić M, Djakovic L, Šujić R, Godman B, Janković SM. Inflammatory bowel diseases (Crohn's disease and ulcerative colitis): cost of treatment in Serbia and the implications. Appl Health Econ Health Policy. 2017;15(1):85-93. doi:10.1007/s40258-016-0272-z

3. Ferlay J, Soerjomataram I, Dikshit R, et al. Cancer incidence and mortality worldwide: sources, methods and major patterns in GLOBOCAN 2012. Int $J$ Cancer. 2015;136(5):E359-86. doi:10.1002/ijc. 29210

4. Burisch J, Munkholm P. The epidemiology of inflammatory bowel disease. Scand J Gastroenterol. 2015;50(8):942-951. doi:10.3109/00365521.2015.1014407

5. Burisch J, Jess T, Martinato M, Lakatos PL; ECCO-EpiCom. The burden of inflammatory bowel disease in Europe. J Crohns Colitis. 2013;7(4):322-337. doi:10.1016/j.crohns.2013.01.010

6. Kaplan GG, Bernstein CN, Coward S, et al. The impact of inflammatory bowel disease in Canada 2018: epidemiology. J Can Assoc Gastroenterol. 2019;2(Suppl 1):S6-s16. doi:10.1093/jcag/gwy054

7. M'koma AE. Inflammatory bowel disease: an expanding global health problem. Clin Med Insights Gastroenterol. 2013;6(CGast): S12731.

8. Burisch J. Crohn's disease and ulcerative colitis. Occurrence, course and prognosis during the first year of disease in a European population-based inception cohort. Dan Med J. 2014;61(1):B4778.

9. Itzkowitz SH, Yio X. Inflammation and Cancer IV. Colorectal cancer in inflammatory bowel disease: the role of inflammation. Am J Physiol Gastrointest Liver Physiol. 2004;287(1):G7-G17. doi:10.1152/ajpgi.00079.2004

10. Han W, Xie B, Li Y, et al. Orally deliverable nanotherapeutics for the synergistic treatment of colitis-associated colorectal cancer. Theranostics. 2019;9(24):7458-7473. doi:10.7150/thno.38081

11. Zhang Z, Dong L, Jia A, et al. Glucocorticoids promote the onset of acute experimental colitis and cancer by upregulating mTOR signaling in intestinal epithelial cells. Cancers (Basel). 2020;12 (4):945. doi:10.3390/cancers 12040945

12. Pu J, Zhou X, Liu J, Hou P, Ji M. The therapeutic potential and deleterious effect of glucocorticoids on AOM/DSS-induced colorectal cancer in mice. 2021.

13. Dayharsh GA, Loftus EV, Sandborn WJ, et al. Epstein-Barr viruspositive lymphoma in patients with inflammatory bowel disease treated with azathioprine or 6-mercaptopurine. Gastroenterology. 2002;122(1):72-77. doi:10.1053/gast.2002.30328

14. Talley NJ, Abreu MT, Achkar J-P, et al.; for the American College of Gastroenterology, I. B. D. Task Force. An evidence-based systematic review on medical therapies for inflammatory bowel disease. Am Coll Gastroenterol. 2011;106.

15. Coco R, Plapied L, Pourcelle V, et al. Drug delivery to inflamed colon by nanoparticles: comparison of different strategies. Int $J$ Pharm. 2013;440(1):3-12. doi:10.1016/j.ijpharm.2012.07.017

16. Lautenschläger C, Schmidt C, Fischer D, Stallmach A. Drug delivery strategies in the therapy of inflammatory bowel disease. Adv Drug Deliv Rev. 2014;71:58-76. doi:10.1016/j. addr.2013.10.001
17. Hua S. Orally administered liposomal formulations for colon targeted drug delivery. Front Pharmacol. 2014;5:138. doi:10.3389/fphar.2014.00138

18. Zhang S, Ermann J, Succi MD, et al. An inflammation-targeting hydrogel for local drug delivery in inflammatory bowel disease. Sci Transl Med. 2015;7(300):300ra128-300ra128. doi:10.1126/ scitranslmed.aaa5657

19. Talekar M, Tran T-H, Amiji M. Translational nano-medicines: targeted therapeutic delivery for cancer and inflammatory diseases. AAPS J. 2015;17(4):813-827. doi:10.1208/s12248-0159772-2

20. Yang C, Merlin D. Can naturally occurring nanoparticle-based targeted drug delivery effectively treat inflammatory bowel disease? Expert Opin Drug Deliv. 2020;17(1):1-4. doi:10.1080/ 17425247.2020.1698543

21. Viscido A, Capannolo A, Latella G, Caprilli R, Frieri G. Nanotechnology in the treatment of inflammatory bowel diseases. J Crohns Colitis. 2014;8(9):903-918. doi:10.1016/j. crohns.2014.02.024

22. Ramos GP, Papadakis KA. Mechanisms of Disease: Inflammatory Bowel Diseases, Mayo Clinic Proceedings. Elsevier; 2019:155-165.

23. Hua S, Marks E, Schneider JJ, Keely S. Advances in oral nano-delivery systems for colon targeted drug delivery in inflammatory bowel disease: selective targeting to diseased versus healthy tissue. Nanomedicine. 2015;11(5):1117-1132. doi:10.1016/j.nano.2015.02.018

24. Vass P, Demuth B, Hirsch E, et al. Drying technology strategies for colon-targeted oral delivery of biopharmaceuticals. J Control Release. 2019;296:162-178. doi:10.1016/j.jconrel.2019.01.023

25. Coward S, Clement F, Benchimol EI, et al. Past and future burden of inflammatory bowel diseases based on modeling of population-based data. Gastroenterology. 2019;156(5):13451353. e4. doi:10.1053/j.gastro.2019.01.002

26. Jacob EM, Borah A, Pillai SC, Kumar DS. Inflammatory bowel disease: the emergence of new trends in lifestyle and nanomedicine as the modern tool for pharmacotherapy. Nanomaterials. 2020;10(12):2460. doi:10.3390/nano10122460

27. Guan Q. A comprehensive review and update on the pathogenesis of inflammatory bowel disease. J Immunol Res. 2019;2019:1-16. doi:10.1155/2019/7247238

28. Neurath MF. Targeting immune cell circuits and trafficking in inflammatory bowel disease. Nat Immunol. 2019;20(8):970-979. doi:10.1038/s41590-019-0415-0

29. Kmieć Z, Cyman M, Ślebioda TJ. Cells of the innate and adaptive immunity and their interactions in inflammatory bowel disease. Adv Med Sci. 2017;62(1):1-16. doi:10.1016/j.advms.2016.09.001

30. Sayed IM, Suarez K, Lim E, et al. Host engulfment pathway controls inflammation in inflammatory bowel disease. FEBS $J$. 2020;287(18):3967-3988. doi:10.1111/febs.15236

31. Neurath MF. Cytokines in inflammatory bowel disease. Nat Rev Immunol. 2014;14(5):329-342. doi:10.1038/nri3661

32. Glocker E, Grimbacher B. Inflammatory bowel disease: is it a primary immunodeficiency? Cell Mol Life Sci. 2012;69 (1):41-48. doi:10.1007/s00018-011-0837-9

33. Weisshof R, El Jurdi K, Zmeter N, Rubin DT. Emerging therapies for inflammatory bowel disease. Adv Ther. 2018;35 (11):1746-1762. doi:10.1007/s12325-018-0795-9

34. Katsanos KH, Papadakis KA. Inflammatory bowel disease: updates on molecular targets for biologics. Gut Liver. 2017;11 (4):455. doi:10.5009/gnl16308

35. Singh D, Srivastava S, Pradhan M, Kanwar JR, Singh MR. Inflammatory bowel disease: pathogenesis, causative factors, issues, drug treatment strategies, and delivery approaches. Crit Rev Ther Drug Carrier Syst. 2015;32(3):181-214. doi:10.1615/ CritRevTherDrugCarrierSyst.2015011095 
36. Sales-Campos H, Basso PJ, Alves VB, et al. Classical and recent advances in the treatment of inflammatory bowel diseases. Braz $J$ Med Biol Res. 2015;48(2):96-107. doi:10.1590/1414$431 \times 20143774$

37. Vindigni SM, Zisman TL, Suskind DL, Damman CJ. The intestinal microbiome, barrier function, and immune system in inflammatory bowel disease: a tripartite pathophysiological circuit with implications for new therapeutic directions. Therap Adv Gastroenterol. 2016;9(4):606-625. doi:10.1177/ 1756283 X16644242

38. Waljee AK, Lipson R, Wiitala WL, et al. Predicting hospitalization and outpatient corticosteroid use in inflammatory bowel disease patients using machine learning. Inflamm Bowel Dis. 2018;24(1):45-53. doi:10.1093/ibd/izx007

39. Narula N, Borges L, Steinhart AH, Colombel J-F. Trends in narcotic and corticosteroid prescriptions in patients with inflammatory bowel disease in the United States ambulatory care setting from 2003 to 2011. Inflamm Bowel Dis. 2017;23(6):868-874. doi:10.1097/MIB.0000000000001084

40. Bermejo F, Algaba A, López-Durán S, et al. Mercaptopurine and inflammatory bowel disease: the other thiopurine. Revista Española de Enfermedades Digestivas. 2017;109(1):10-16. doi:10.17235/reed.2016.4546/2016

41. Nielsen $\mathrm{OH}$, Steenholdt C, Juhl CB, Rogler G. Efficacy and safety of methotrexate in the management of inflammatory bowel disease: a systematic review and meta-analysis of randomized, controlled trials. EClinicalMedicine. 2020;20:100271. doi:10.1016/j.eclinm.2020.100271

42. Roblin X, Boschetti G, Williet N, et al. Azathioprine dose reduction in inflammatory bowel disease patients on combination therapy: an openlabel, prospective and randomised clinical trial. Aliment Pharmacol Ther. 2017;46(2):142-149. doi:10.1111/apt.14106

43. Weissman S, Chris-Olaiya A, Mehta TI, et al. A novel player: cyclosporine therapy in the management of inflammatory bowel disease. Transl Gastroenterol Hepatol. 2019;4:67. doi:10.21037/ $\operatorname{tgh} .2019 .08 .08$

44. van de Meeberg MM, Schultheiss JP, Oldenburg B, Fidder HH, Huitema AD. Does the 5-aminosalicylate concentration correlate with the efficacy of oral 5-aminosalicylate and predict response in patients with inflammatory bowel disease? A systematic review. Digestion. 2020;101(3):245-261. doi:10.1159/000499331

45. Ma H-Q, Yu -T-T, Zhao X-J, Zhang Y, Zhang H-J. Fecal microbial dysbiosis in Chinese patients with inflammatory bowel disease. World J Gastroenterol. 2018;24(13):1464. doi:10.3748/ wjg.v24.i13.1464

46. Di Paolo A, Luci G. Personalized medicine of monoclonal antibodies in inflammatory bowel disease: pharmacogenetics, therapeutic drug monitoring, and beyond. Front Pharmacol. 2020;11. doi:10.3389/fphar.2020.610806

47. Sheasgreen C, Nguyen GC. The evolving evidence for therapeutic drug monitoring of monoclonal antibodies in inflammatory bowel disease. Curr Gastroenterol Rep. 2017;19(5):19. doi:10.1007/ s11894-017-0559-8

48. Rawla P, Sunkara T, Raj JP. Role of biologics and biosimilars in inflammatory bowel disease: current trends and future perspectives. J Inflamm Res. 2018;11:215. doi:10.2147/JIR. S165330

49. De Vries L, Wildenberg M, De Jonge W, D’Haens GR. The future of Janus kinase inhibitors in inflammatory bowel disease. J Crohn's Colitis. 2017;11(7):885-893. doi:10.1093/ecco-jcc /jjx003

50. Christensen B, Gibson PR, Micic D, et al. Safety and efficacy of combination treatment with calcineurin inhibitors and vedolizumab in patients with refractory inflammatory bowel disease. Clin Gastroenterol Hepatol. 2019;17(3):486-493. doi:10.1016/j. cgh.2018.04.060
51. Papamichael K, Lin S, Moore M, Papaioannou G, Sattler L, Cheifetz AS. Infliximab in inflammatory bowel disease. Ther Adv Chronic Dis. 2019;10:2040622319838443. doi:10.1177/ 2040622319838443

52. Barré A, Colombel JF, Ungaro R. predictors of response to vedolizumab and ustekinumab in inflammatory bowel disease. Aliment Pharmacol Ther. 2018;47(7):896-905. doi:10.1111/apt.14550

53. Amiot A, Serrero M, Peyrin-Biroulet L, et al. One-year effectiveness and safety of vedolizumab therapy for inflammatory bowel disease: a prospective multicentre cohort study. Aliment Pharmacol Ther. 2017;46(3):310-321. doi:10.1111/apt.14167

54. Dayan JR, Dolinger M, Benkov K, et al. Real world experience with ustekinumab in children and young adults at a tertiary care pediatric inflammatory bowel disease center. $J$ Pediatr Gastroenterol Nutr. 2019;69(1):61. doi:10.1097/MPG.000000 0000002362

55. Olivera P, Danese S, Peyrin-Biroulet L. JAK inhibition in inflammatory bowel disease. Expert Rev Clin Immunol. 2017;13 (7):693-703. doi:10.1080/1744666X.2017.1291342

56. Biswas S, Bryant RV, Travis S. Interfering with leukocyte trafficking in Crohn's disease. Best Pract Res Clin Gastroenterol. 2019;38:101617. doi:10.1016/j.bpg.2019.05.004

57. Mao F, Tu Q, Wang L, et al. Mesenchymal stem cells and their therapeutic applications in inflammatory bowel disease. Oncotarget. 2017;8(23):38008. doi:10.18632/oncotarget.16682

58. Holmberg FE, Pedersen J, Jørgensen P, Soendergaard C, Jensen $\mathrm{KB}$, Nielsen $\mathrm{OH}$. Intestinal barrier integrity and inflammatory bowel disease: stem cell-based approaches to regenerate the barrier. J Tissue Eng Regen Med. 2018;12(4):923-935. doi:10.1002/term.2506

59. Tsuchiya A, Kojima Y, Ikarashi S, et al. Clinical trials using mesenchymal stem cells in liver diseases and inflammatory bowel diseases. Inflamm Regen. 2017;37(1):1-15. doi:10.1186/ s41232-017-0045-6

60. Pouya S, Heidari M, Baghaei K, et al. Study the effects of mesenchymal stem cell conditioned medium injection in mouse model of acute colitis. Int Immunopharmacol. 2018;54:86-94. doi:10.1016/j.intimp.2017.11.001

61. Holmberg FE, Seidelin JB, Yin X, et al. Culturing human intestinal stem cells for regenerative applications in the treatment of inflammatory bowel disease. EMBO Mol Med. 2017;9 (5):558-570. doi:10.15252/emmm.201607260

62. Zhang S, Langer R, Traverso G. Nanoparticulate drug delivery systems targeting inflammation for treatment of inflammatory bowel disease. Nano Today. 2017;16:82-96. doi:10.1016/j. nantod.2017.08.006

63. Guo Y, Zong S, Pu Y, Xu B, Zhang T, Wang B. Advances in pharmaceutical strategies enhancing the efficiencies of oral colon-targeted delivery systems in inflammatory bowel disease. Molecules. 2018;23(7):1622. doi:10.3390/molecules23071622

64. Kotla NG, Rana S, Sivaraman G, et al. Bioresponsive drug delivery systems in intestinal inflammation: state-of-the-art and future perspectives. Adv Drug Deliv Rev. 2019;146:248-266. doi:10.1016/j.addr.2018.06.021

65. Lamprecht A, Schäfer U, Lehr C-M. Size-dependent bioadhesion of micro-and nanoparticulate carriers to the inflamed colonic mucosa. Pharm Res. 2001;18(6):788-793. doi:10.1023/ A:1011032328064

66. Wang L, Yu M, Yang H. Recent progress in the diagnosis and precise nanocarrier-mediated therapy of inflammatory bowel disease. J Inflamm Res. 2021;14:1701-1716. doi:10.2147/JIR. S304101

67. Youshia J, Lamprecht A. Size-dependent nanoparticulate drug delivery in inflammatory bowel diseases. Expert Opin Drug Deliv. 2016;13(2):281-294. doi:10.1517/17425247.2016.1114 604 
68. Song W, Shen L, Wang Y, et al. Synergistic and low adverse effect cancer immunotherapy by immunogenic chemotherapy and locally expressed PD-L1 trap. Nat Commun. 2018;9(1):2237. doi:10.1038/s41467-018-04605-x

69. Xiao B, Merlin D. Oral colon-specific therapeutic approaches toward treatment of inflammatory bowel disease. Expert Opin Drug Deliv. 2012;9(11):1393-1407. doi:10.1517/17425247.2012.730517

70. Sun T, Kwong CHT, Gao C, et al. Amelioration of ulcerative colitis via inflammatory regulation by macrophage-biomimetic nanomedicine. Theranostics. 2020;10(22):10106-10119. doi: $10.7150 /$ thno. 48448

71. Chung CH, Jung W, Keum H, Kim TW, Jon S. Nanoparticles derived from the natural antioxidant rosmarinic acid ameliorate acute inflammatory bowel disease. ACS Nano. 2020;14 (6):6887-6896. doi:10.1021/acsnano.0c01018

72. Miao L, Guo S, Lin CM, Liu Q, Huang L. Nanoformulations for combination or cascade anticancer therapy. Adv Drug Deliv Rev. 2017;115:3-22. doi:10.1016/j.addr.2017.06.003

73. Qiu N, Gao J, Liu Q, Wang J, Shen Y. Enzyme-responsive charge-reversal polymer-mediated effective gene therapy for intraperitoneal tumors. Biomacromolecules. 2018;19 (6):2308-2319. doi:10.1021/acs.biomac.8b00440

74. Chen Y, Song W, Shen L, et al. Vasodilator hydralazine promotes nanoparticle penetration in advanced desmoplastic tumors. ACS Nano. 2019;13(2):1751-1763. doi:10.1021/acsnano.8b07830

75. Cuvelier CA, Quatacker J, Mielants H, Vos MD, Veys E, Roels HJ. M-cells are damaged and increased in number in inflamed human ileal mucosa. Histopathology. 1994;24 (5):417-426. doi:10.1111/j.1365-2559.1994.tb00550.x

76. Pichai MV, Ferguson LR. Potential prospects of nanomedicine for targeted therapeutics in inflammatory bowel diseases. World J Gastroenterol. 2012;18(23):2895. doi:10.3748/wjg.v18.i23.2895

77. Colombel J-F, Narula N, Peyrin-Biroulet L. Management strategies to improve outcomes of patients with inflammatory bowel diseases. Gastroenterology. 2017;152(2):351-361. e5. doi:10.1053/j.gastro.2016.09.046

78. Mohan LJ, Daly JS, Ryan BM, Ramtoola Z. The future of nanomedicine in optimising the treatment of inflammatory bowel disease. Scand J Gastroenterol. 2019;54(1):18-26. doi:10.1080/ 00365521.2018 .1563805

79. Yin Y, Yang J, Pan Y, et al. Mesopore to macropore transformation of metal-organic framework for drug delivery in inflammatory bowel disease. Adv Healthc Mater. 2021;10(3):e2000973. doi:10.1002/adhm.202000973

80. Sharpe LA, Daily AM, Horava SD, Peppas NA. Therapeutic applications of hydrogels in oral drug delivery. Expert Opin Drug Deliv. 2014;11(6):901-915. doi:10.1517/17425247.2014.902047

81. Deshmukh R, Harwansh RK, Paul SD, Shukla R. Controlled release of sulfasalazine loaded amidated pectin microparticles through Eudragit S 100 coated capsule for management of inflammatory bowel disease. J Drug Deliv Sci Technol. 2020;55:101495. doi:10.1016/j.jddst.2019.101495

82. Hou L, Zheng Y, Wang Y, et al. Self-regulated carboxyphenylboronic acid-modified mesoporous silica nanoparticles with "touch switch" releasing property for insulin delivery. ACS Appl Mater Interfaces. 2018;10(26):21927-21938. doi:10.1021/ acsami.8b06998

83. Minakshi P, Kumar R, Ghosh M, Brar B, Barnela M, Lakhani P. Application of polymeric nano-materials in management of inflammatory bowel disease. Curr Top Med Chem. 2020;20 (11):982-1008.

84. Wang Q-S, Wang G-F, Zhou J, Gao L-N, Cui Y-L. Colon targeted oral drug delivery system based on alginate-chitosan microspheres loaded with icariin in the treatment of ulcerative colitis. Int J Pharm. 2016;515 (1-2):176-185. doi:10.1016/j.ijpharm.2016.10.002
85. Oliva N, Conde J, Wang K, Artzi N. Designing hydrogels for on-demand therapy. Acc Chem Res. 2017;50(4):669-679. doi:10.1021/acs.accounts.6b00536

86. Laroui H, Dalmasso G, Nguyen HTT, Yan Y, Sitaraman SV, Merlin D. Drug-loaded nanoparticles targeted to the colon with polysaccharide hydrogel reduce colitis in a mouse model. Gastroenterology. 2010;138(3):843-853. e2. doi:10.1053/j. gastro.2009.11.003

87. Beloqui A, Coco R, Alhouayek M, et al. Budesonide-loaded nanostructured lipid carriers reduce inflammation in murine DSS-induced colitis. Int $J$ Pharm. 2013;454(2):775-783. doi:10.1016/j.ijpharm.2013.05.017

88. Vafaei SY, Esmaeili M, Amini M, Atyabi F, Ostad SN, Dinarvand R. Self assembled hyaluronic acid nanoparticles as a potential carrier for targeting the inflamed intestinal mucosa. Carbohydr Polym. 2016;144:371-381. doi:10.1016/j. carbpol.2016.01.026

89. Dianzani C, Foglietta F, Ferrara B, et al. Solid lipid nanoparticles delivering anti-inflammatory drugs to treat inflammatory bowel disease: effects in an in vivo model. World $J$ Gastroenterol. 2017;23(23):4200. doi:10.3748/wjg.v23.i23.4200

90. Lamprecht A, Yamamoto H, Takeuchi H, Kawashima Y. Nanoparticles enhance therapeutic efficiency by selectively increased local drug dose in experimental colitis in rats. J Pharmacol Exp Therap. 2005;315(1):196-202. doi:10.1124/ jpet.105.088146

91. Muheem A, Shakeel F, Jahangir MA, et al. A review on the strategies for oral delivery of proteins and peptides and their clinical perspectives. Saudi Pharm J. 2016;24(4):413-428. doi:10.1016/j.jsps.2014.06.004

92. Vegter S, Tolley K, Wilson Waterworth T, Jones H, Jones S, Jewell D. Meta-analysis using individual patient data: efficacy and durability of topical alicaforsen for the treatment of active ulcerative colitis. Aliment Pharmacol Ther. 2013;38(3):284-293. doi:10.1111/apt.12369

93. Monteleone G, Neurath MF, Ardizzone S, et al. Mongersen, an oral SMAD7 antisense oligonucleotide, and Crohn's disease. $N$ Engl J Med. 2015;372(12):1104-1113. doi:10.1056/ NEJMoa1407250

94. Collnot E-M, Ali H, Lehr C-M. Nano- and microparticulate drug carriers for targeting of the inflamed intestinal mucosa. J Controll Release. 2012;161(2):235-246. doi:10.1016/j.jconrel.2012.01.028

95. Guo J, Jiang X, Gui S. RNA interference-based nanosystems for inflammatory bowel disease therapy. Int $J$ Nanomedicine. 2016;11:5287-5310. doi:10.2147/IJN.S116902

96. Di Fusco D, Dinallo V, Marafini I, Figliuzzi MM, Romano B, Monteleone G. Antisense oligonucleotide: basic concepts and therapeutic application in inflammatory bowel disease. Front Pharmacol. 2019;10:305. doi:10.3389/fphar.2019.00305

97. Tahara K, Samura S, Tsuji K, et al. Oral nuclear factor- $\kappa B$ decoy oligonucleotides delivery system with chitosan modified poly (D, L-lactide-co-glycolide) nanospheres for inflammatory bowel disease. Biomaterials. 2011;32(3):870-878. doi:10.1016/j. biomaterials.2010.09.034

98. Arthur Kaser S, Richard Blumberg S, Blumberg RS. Inflammatory bowel disease. Annu Rev Immunol. 2010;28:573-621. doi:10.1146/annurev-immunol-030409-101225

99. Verma P, Srivastava A, Srikanth CV, Bajaj A. Nanoparticlemediated gene therapy strategies for mitigating inflammatory bowel disease. Biomater Sci. 2021;9:1481-1502. doi:10.1039/ D0BM01359E

100. Xu X, Yang W, Liang Q, et al. Efficient and targeted drug/siRNA co-delivery mediated by reversibly crosslinked polymersomes toward anti-inflammatory treatment of ulcerative colitis (UC). Nano Res. 2019;12(3):659-667. doi:10.1007/s12274-019-2274-2 
101. Yang C, Zhang M, Lama S, Wang L, Merlin D. Natural-lipid nanoparticle-based therapeutic approach to deliver 6-shogaol and its metabolites M2 and M13 to the colon to treat ulcerative colitis. J Controll Release. 2020;323:293-310. doi:10.1016/j. jconrel.2020.04.032

102. Khare T, Palakurthi SS, Shah BM, Palakurthi S, Khare S. Natural product-based nanomedicine in treatment of inflammatory bowel disease. Int $J$ Mol Sci. 2020;21(11):3956. doi:10.3390/ ijms21113956

103. Li Y, Fan L, Tang T, et al. Modified apple polysaccharide prevents colitis through modulating IL-22 and IL-22BP expression. Int $J$ Biol Macromol. 2017;103:1217-1223. doi:10.1016/j. ijbiomac.2017.05.172

104. Maria-Ferreira D, Nascimento AM, Cipriani TR, et al. Rhamnogalacturonan, a chemically-defined polysaccharide, improves intestinal barrier function in DSS-induced colitis in mice and human Caco-2 cells. Sci Rep. 2018;8(1):12261. doi:10.1038/s41598-018-30526-2

105. Nie Y, Lin Q, Luo F. Effects of non-starch polysaccharides on inflammatory bowel disease. Int J Mol Sci. 2017;18(7):1372 doi:10.3390/ijms 18071372

106. Li J, Chen H, Wang B, et al. ZnO nanoparticles act as supportive therapy in DSS-induced ulcerative colitis in mice by maintaining gut homeostasis and activating Nrf2 signaling. Sci Rep. 2017;7:43126. doi:10.1038/srep43126

107. Bhavsar MD, Amiji MM. Oral IL-10 gene delivery in a microsphere-based formulation for local transfection and therapeutic efficacy in inflammatory bowel disease. Gene Ther. 2008;15(17):1200-1209. doi:10.1038/gt.2008.67

108. Kriegel C, Amiji M. Oral TNF- $\alpha$ gene silencing using a polymeric microsphere-based delivery system for the treatment of inflammatory bowel disease. J Control Release. 2011;150 (1):77-86. doi:10.1016/j.jconrel.2010.10.002

109. Castangia I, Nácher A, Caddeo C, et al. Therapeutic efficacy of quercetin enzyme-responsive nanovesicles for the treatment of experimental colitis in rats. Acta Biomaterialia. 2015;13:216-227. doi:10.1016/j.actbio.2014.11.017

110. Shen C, Zhao L, Du X, et al. Smart responsive quercetin-conjugated glycol chitosan prodrug micelles for treatment of inflammatory bowel diseases. Mol Pharm. 2021;18:1419-1430. doi:10.1021/acs.molpharmaceut.0c01245

111. Patole VC, Pandit AP. Mesalamine-loaded alginate microspheres filled in enteric coated HPMC capsules for local treatment of ulcerative colitis: in vitro and in vivo characterization. J Pharm Investig. 2018;48(3):257-267. doi:10.1007/s40005-017-0304-1

112. Wang B, Zhuang X, Deng ZB, et al. Targeted drug delivery to intestinal macrophages by bioactive nanovesicles released from grapefruit. Mol Ther. 2014;22(3):522-534. doi:10.1038/mt.2013.190

113. Zhang M, Viennois E, Prasad M, et al. Edible ginger-derived nanoparticles: a novel therapeutic approach for the prevention and treatment of inflammatory bowel disease and colitis-associated cancer. Biomaterials. 2016;101:321-340. doi:10.1016/j.biomaterials.2016.06.018

114. Zhang M, Xu C, Liu D, Han MK, Wang L, Merlin D. Oral delivery of nanoparticles loaded with ginger active compound, 6-shogaol, attenuates ulcerative colitis and promotes wound healing in a murine model of ulcerative colitis. J Crohns Colitis. 2018;12(2):217-229. doi:10.1093/ecco-jcc/jjx115

115. Zhu C, Zhang S, Song C, et al. Selenium nanoparticles decorated with Ulva lactuca polysaccharide potentially attenuate colitis by inhibiting NF-кB mediated hyper inflammation. J Nanobiotechnology. 2017;15 (1):20. doi:10.1186/s12951-017-0252-y

116. Deng Z, Rong Y, Teng Y, et al. Broccoli-derived nanoparticle inhibits mouse colitis by activating dendritic cell AMP-activated protein kinase. Mol Ther. 2017;25(7):1641-1654. doi:10.1016/j. ymthe.2017.01.025
117. Kim J-H, Hong -S-S, Lee M, et al. Krill oil-incorporated liposomes as an effective nanovehicle to ameliorate the inflammatory responses of DSS-induced colitis. Int $J$ Nanomedicine. 2019;14:8305. doi:10.2147/IJN.S220053

118. Chambers E, Mitragotri S. Prolonged circulation of large polymeric nanoparticles by non-covalent adsorption on erythrocytes. $J$ Control Release. 2004;100(1):111-119. doi:10.1016/j. jconrel.2004.08.005

119. Markov DE, Boeve H, Gleich B, et al. Human erythrocytes as nanoparticle carriers for magnetic particle imaging. Phys Med Biol. 2010;55(21):6461-6473. doi:10.1088/0031-9155/55/21/008

120. Castro M, Rossi L, Papadatou B, et al. Long-term treatment with autologous red blood cells loaded with dexamethasone 21-phosphate in pediatric patients affected by steroid-dependent Crohn disease. J Pediatr Gastroenterol Nutr. 2007;44 (4):423-426. doi:10.1097/MPG.0b013e3180320667

121. Papadatou B, Knafelz D, Bracci F, et al. P351 Long term treatment with autologous red blood cells loaded with dexamethasone 21-phosphate in pediatric patients affected by steroid-dependent Crohn's disease: in the era of biologics can steroids be reconsidered? J Crohns Colitis. 2012;6(Supplement_1):S149-S149. doi:10.1016/S1873-9946(12)60370-5

122. Li X, Lu C, Yang Y, Yu C, Rao Y. Site-specific targeted drug delivery systems for the treatment of inflammatory bowel disease. Biomed Pharmacother. 2020;129:110486. doi:10.1016/j. biopha.2020.110486

123. Ha D, Yang N, Nadithe V. Exosomes as therapeutic drug carriers and delivery vehicles across biological membranes: current perspectives and future challenges. Acta Pharmaceutica Sinica B. 2016;6(4):287-296. doi:10.1016/j.apsb.2016.02.001

124. Baghaei K, Tokhanbigli S, Asadzadeh H, Nmaki S, Reza Zali M, Hashemi SM. Exosomes as a novel cell-free therapeutic approach in gastrointestinal diseases. $J$ Cell Physiol. 2019;234 (7):9910-9926. doi:10.1002/jcp.27934

125. Di Gioia S, Hossain MN, Conese M. Biological properties and therapeutic effects of plant-derived nanovesicles. Open Med (Wars). 2020;15(1):1096-1122. doi:10.1515/med-2020-0160

126. Van Niel G, Mallegol J, Bevilacqua C, et al. Intestinal epithelial exosomes carry MHC class II/peptides able to inform the immune system in mice. Gut. 2003;52(12):1690-1697. doi:10.1136/ gut.52.12.1690

127. Wang Y, Tian J, Tang X, et al. Exosomes released by granulocytic myeloid-derived suppressor cells attenuate DSS-induced colitis in mice. Oncotarget. 2016;7(13):15356. doi:10.18632/oncotarget.7324

128. Yang J, Liu -X-X, Fan H, et al. Extracellular vesicles derived from bone marrow mesenchymal stem cells protect against experimental colitis via attenuating colon inflammation, oxidative stress and apoptosis. PLoS One. 2015;10(10):e0140551. doi:10.1371/journal.pone.0140551

129. Mao F, Wu Y, Tang X, et al. Exosomes derived from human umbilical cord mesenchymal stem cells relieve inflammatory bowel disease in mice. Biomed Res Int. 2017;2017:1-12. doi:10.1155/2017/5356760

130. Wu Y, Qiu W, Xu X, et al. Exosomes derived from human umbilical cord mesenchymal stem cells alleviate inflammatory bowel disease in mice through ubiquitination. Am J Transl Res. 2018;10(7):2026

131. Gao J-G, Yu M-S, Zhang -M-M, et al. Adipose-derived mesenchymal stem cells alleviate TNBS-induced colitis in rats by influencing intestinal epithelial cell regeneration, Wnt signaling, and T cell immunity. World J Gastroenterol. 2020;26(26):3750. doi:10.3748/wjg.v26.i26.3750

132. Liu P, Gao C, Chen H, et al. Receptor-mediated targeted drug delivery systems for treatment of inflammatory bowel disease: opportunities and emerging strategies. Acta Pharmaceutica Sinica B. 2020. doi:10.1016/j.apsb.2020.11.003 
133. Kim E, Schueller O, Sweetnam PM. Targeting the leukocyte activation cascade: getting to the site of inflammation using microfabricated assays. Lab Chip. 2012;12(12):2255-2264. doi:10.1039/c2lc21078a

134. Cox D, Brennan M, Moran N. Integrins as therapeutic targets: lessons and opportunities. Nat Rev Drug Discov. 2010;9 (10):804-820. doi:10.1038/nrd3266

135. Farkas S, Hornung M, Sattler C, et al. Short-term treatment with anti-CD44v7 antibody, but not CD44v4, restores the gut mucosa in established chronic dextran sulphate sodium (DSS)-induced colitis in mice. Clin Exp Immunol. 2005;142(2):260-267. doi:10.1111/j.1365-2249.2005.02911.x

136. Dreaden EC, Morton SW, Shopsowitz KE, et al. Bimodal tumor-targeting from microenvironment responsive hyaluronan Layer-by-Layer (LbL) nanoparticles. ACS Nano. 2014;8 (8):8374-8382. doi:10.1021/nn502861t

137. Xiao B, Xu Z, Viennois E, et al. Orally targeted delivery of tripeptide KPV via hyaluronic acid-functionalized nanoparticles efficiently alleviates ulcerative colitis. Mol Ther. 2017;25 (7):1628-1640. doi:10.1016/j.ymthe.2016.11.020

138. Chapkin RS, Kamen BA, Callaway ES, et al. Use of a novel genetic mouse model to investigate the role of folate in colitis-associated colon cancer. J Nutr Biochem. 2009;20 (8):649-655. doi:10.1016/j.jnutbio.2008.07.001

139. Spencer SP, Belkaid Y. Dietary and commensal derived nutrients: shaping mucosal and systemic immunity. Curr Opin Immunol. 2012;24(4):379-384. doi:10.1016/j.coi.2012.07.006

140. Günter EA, Markov PA, Melekhin AK, et al. Preparation and release characteristics of mesalazine loaded calcium pectin-silica gel beads based on callus cultures pectins for colon-targeted drug delivery. Int J Biol Macromol. 2018;120(Pt B):2225-2233. doi:10.1016/j.ijbiomac.2018.07.078

141. Kriegel C, Amiji MM. Dual TNF- $\alpha$ /cyclin D1 gene silencing with an oral polymeric microparticle system as a novel strategy for the treatment of inflammatory bowel disease. Clin Transl Gastroenterol. 2011;2(3):e2-e2. doi:10.1038/ctg.2011.1

142. Xiao B, Yang Y, Viennois E, et al. Glycoprotein CD98 as a receptor for colitis-targeted delivery of nanoparticles. J Mater Chem B. 2014;2(11):1499-1508. doi:10.1039/c3tb21564d

143. Laroui H, Geem D, Xiao B, et al. Targeting intestinal inflammation with CD98 siRNA/PEI-loaded nanoparticles. Mol Ther. 2014;22(1):69-80. doi:10.1038/mt.2013.214

144. Nguyen HTT, Dalmasso G, Torkvist L, et al. CD98 expression modulates intestinal homeostasis, inflammation, and colitis-associated cancer in mice. J Clin Invest. 2011;121 (5):1733-1747. doi:10.1172/JCI44631

145. Iglesias N, Galbis E, Díaz-Blanco MJ, Lucas R, Benito E, De-paz MV. Nanostructured chitosan-based biomaterials for sustained and colon-specific resveratrol release. Int J Mol Sci. 2019;20 (2):398. doi:10.3390/ijms20020398

146. Makhlof A, Tozuka Y, Takeuchi H. pH-Sensitive nanospheres for colon-specific drug delivery in experimentally induced colitis rat model. Eur J Pharm Biopharm. 2009;72(1):1-8. doi:10.1016/j. ejpb.2008.12.013
147. Sun Q, Luan L, Arif M, et al. Redox-sensitive nanoparticles based on 4-aminothiophenol-carboxymethyl inulin conjugate for budesonide delivery in inflammatory bowel diseases. Carbohydr Polym. 2018;189:352-359. doi:10.1016/j.carbpol.2017.12.021

148. Simmonds NJ, Allen RE, Stevens TRJ, et al. Chemiluminescence assay of mucosal reactive oxygen metabolites in inflammatory bowel disease. Gastroenterology. 1992;103(1):186-196. doi:10.1016/0016-5085(92)91112-H

149. Sedghi S, Fields JZ, Klamut M, et al. Increased production of luminol enhanced chemiluminescence by the inflamed colonic mucosa in patients with ulcerative colitis. Gut. 1993;34(9):1191. doi:10.1136/gut.34.9.1191

150. Vong LB, Tomita T, Yoshitomi T, Matsui H, Nagasaki Y. An orally administered redox nanoparticle that accumulates in the colonic mucosa and reduces colitis in mice. Gastroenterology. 2012;143(4):1027-1036. e3. doi:10.1053/j.gastro.2012.06.043

151. Zhang Q, Tao H, Lin Y, et al. A superoxide dismutase/catalase mimetic nanomedicine for targeted therapy of inflammatory bowel disease. Biomaterials. 2016;105:206-221. doi:10.1016/j. biomaterials.2016.08.010

152. Wilson DS, Dalmasso G, Wang L, Sitaraman SV, Merlin D, Murthy N. Orally delivered thioketal nanoparticles loaded with TNF- $\alpha-$ siRNA target inflammation and inhibit gene expression in the intestines. Nat Mater. 2010;9(11):923-928. doi:10.1038/nmat2859

153. Antoni L, Nuding S, Wehkamp J, Stange EF. Intestinal barrier in inflammatory bowel disease. World J Gastroenterol. 2014;20 (5):1165-1179. doi:10.3748/wjg.v20.i5.1165

154. Ma TY, Boivin MA, Ye D, Pedram A, Said HM. Mechanism of TNF- $\alpha$ modulation of Caco-2 intestinal epithelial tight junction barrier: role of myosin light-chain kinase protein expression. Am J Physiol Gastrointest Liver Physiol. 2005;288(3):G422-G430. doi:10.1152/ajpgi.00412.2004

155. Schmidt C, Lautenschlaeger C, Collnot E-M, et al. Nano- and microscaled particles for drug targeting to inflamed intestinal mucosa-A first in vivo study in human patients. $J$ Controll Release. 2013;165(2):139-145. doi:10.1016/j.jconrel.2012.10.019

156. Lautenschläger C, Schmidt C, Lehr C-M, Fischer D, Stallmach A. PEG-functionalized microparticles selectively target inflamed mucosa in inflammatory bowel disease. Eur J Pharm Biopharm. 2013;85(3):578-586. doi:10.1016/j.ejpb.2013.09.016

157. Jubeh TT, Barenholz Y, Rubinstein A. Differential adhesion of normal and inflamed rat colonic mucosa by charged liposomes. Pharm Res. 2004;21(3):447-453. doi:10.1023/B: PHAM.0000019298.29561.cd

158. Liu Q, Chen F, Hou L, et al. Nanocarrier-mediated chemo-immunotherapy arrested cancer progression and induced tumor dormancy in desmoplastic melanoma. ACS Nano. 2018;12 (8):7812-7825. doi:10.1021/acsnano.8b01890

159. Mitchell MJ, Billingsley MM, Haley RM, Wechsler ME, Peppas NA, Langer R. Engineering precision nanoparticles for drug delivery. Nat Rev Drug Discov. 2021;20(2):101-124.

160. Lamas B, Martins Breyner N, Houdeau E. Impacts of foodborne inorganic nanoparticles on the gut microbiota-immune axis: potential consequences for host health. Part Fibre Toxicol. 2020;17(1): 19 . 


\section{Publish your work in this journal}

The International Journal of Nanomedicine is an international, peerreviewed journal focusing on the application of nanotechnology in diagnostics, therapeutics, and drug delivery systems throughout the biomedical field. This journal is indexed on PubMed Central, MedLine, CAS, SciSearch ${ }^{\mathbb{B}}$, Current Contents ${ }^{\mathbb{B}} /$ Clinical Medicine,
Journal Citation Reports/Science Edition, EMBase, Scopus and the Elsevier Bibliographic databases. The manuscript management system is completely online and includes a very quick and fair peer-review system, which is all easy to use. Visit http://www.dovepress.com/ testimonials.php to read real quotes from published authors. 